Check for updates

Cite this: RSC Adv., 2019, 9, 38902

\title{
Three cobalt-based coordination polymers with tripodal carboxylate and imidazole-containing ligands: syntheses, structures, properties and DFT studies $\dagger$
}

\author{
Long Tang, (D) Huan-Huan Wang, Yu-Hao Fu, Yi-Tong Wang, JiJiang Wang (D) * \\ and XiangYang $\mathrm{Hou}$
}

Three cobalt-based coordination polymers [Co(Htatb)(1,3-bimyb)] (1), [Co(Htatb)(bimbp)].DMF (2), and

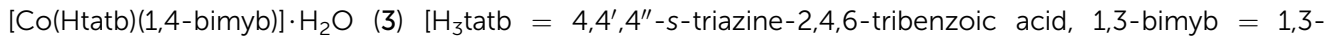
bis(imidazole-1-ylmethyl)benzene, bimbp =4,4'-bis(imidazolyl)biphenyl, 1,4-bimyb =1,4-bis (imidazole1-ylmethyl)benzene] were synthesized by hydrothermal reactions and characterized by single-crystal $X$ ray diffraction, thermogravimetric analyses, IR spectroscopy, UV-vis spectroscopy and elemental analysis. Compound 1 shows a double-strand chain structure, due to the intermolecular $\mathrm{O}-\mathrm{H} \cdots \mathrm{O}$ hydrogen bonds and aromatic $\pi-\pi$ stacking interactions, the adjacent chains are connected to produce a 3D supramolecular structure. Compound 2 shows a 2D structure with a 1D channel. Compound 3 displays a 2D layer structure, furthermore these layers are joined by $\mathrm{O}-\mathrm{H} \cdots \mathrm{O}$ hydrogen bonding to generate a four-fold interpenetrating 3D architecture. The fluorescence properties of 1-3 and the magnetic behavior of 1 and 2 have also been investigated. Based on their crystal structures, compounds 1 and 2 were investigated using hybrid DFT methods at the B3LYP/6-31G (d) level. The DFT-BS approach was applied to study the magnetic coupling behavior. The results reveal that the calculated exchange coupling constants $\mathrm{J}$ were in good agreement with the experimental data.

Received 24th September 2019 Accepted 20th November 2019

DOI: 10.1039/c9ra07737e

rsc.li/rsc-advances solvents, temperature, $\mathrm{pH}$ value, and other factors, have been validated and summarized. ${ }^{9-12}$ The mixed-ligand strategy by the judicious choice of various organic linkers has been proven to be highly-efficient for the construction of metalorganic coordination polymers. Among such systems, most outstanding is the incorporation of polycarboxylates and $\mathrm{N}$ donor co-ligands, ${ }^{13-15}$ which has successfully been utilized to generate diverse and interesting polymeric networks with potential properties and contributes to refining our knowledge of self-assembly processes. Within polycarboxylate ligands, aromatic polycarboxyl compounds have extensively been documented as multifunctional tectons, owing to their versatile linking capability in virtue of both covalent bonding and supramolecular interactions. ${ }^{16-18}$

In our strategy, multidentate $\mathrm{O}-$ or $\mathrm{N}$-donor ligands have also been employed in the construction of coordination polymers. Among the family of organic carboxylate, tripodal carboxylate shows more superiority, ${ }^{19-21}$ the ligand $4,4^{\prime}, 4^{\prime \prime}$-s-triazine-2,4,6tribenzoic acid $\left(\mathrm{H}_{3} \mathrm{tatb}\right)$ as a class example of tripodal ligands has been utilized, and some metal-organic coordination polymers based on $\mathrm{H}_{3}$ tatb have also been investigated. ${ }^{22-28}$ However, metalorganic coordination polymers based on $\mathrm{H}_{3}$ tatb and $\mathrm{N}$-donor ligands have only been investigated scarcely. ${ }^{29}$ To explore the influence of $\mathrm{N}$-donor ligands on achieving different dimensional 
and topological structures based on tripodal carboxylate ligand, we also employ 1,3-bis(imidazole-1-ylmethyl)benzene(1,3-bimyb), 4,4' bis(imidazolyl)biphenyl(bimbp), and 1,4-bis(imidazole-1-ylmethyl) benzene(1,4-bimyb) with different conformations as co-ligands. Three cobalt-based coordination polymers [Co(Htatb)(1,3-bimyb)] (1), [Co(Htatb)(bimbp)]·DMF (2), and [Co(Htatb)(1,4-bimyb)] $\mathrm{H}_{2} \mathrm{O}$ (3) were synthesized and characterized. In addition, their fluorescent properties and magnetic behavior were also investigated in this paper.

\section{Experimental sections}

\subsection{Materials and chemical analysis}

The $\mathrm{H}_{3}$ tatb, 1,3-bimyb, bimbp and 1,4-bimyb ligands were purchased in the Jinan Henghua Sci. \& Tec. Co., Ltd.; all other reagents and solvents employed were commercially available and used without further purification. Elemental analyses were performed with a PerkinElmer $2400 \mathrm{CHN}$ Elemental analyzer. Infrared spectra on $\mathrm{KBr}$ pellets were recorded on a Nicolet 170SX FT-IR spectrophotometer in the range $400-4000 \mathrm{~cm}^{-1}$. UV-vis spectra were recorded on a Shimadzu UV-3600 spectrophotometer in the region $200-700 \mathrm{~nm}$. TG analyses were conducted with a Nietzsch STA 449C micro analyzer under atmosphere at a heating rate of $5{ }^{\circ} \mathrm{C} \min ^{-1}$. X-ray powder diffraction (PXRD) patterns were recorded on a Shimadzu XRD7000 diffractometer analyzer. The magnetic susceptibilities were obtained on crystalline samples using a Quantum Design MPMS SQUID magnetometer. The fluorescence spectra were studied using a Hitachi F-7100 fluorescence spectrophotometer at room temperature.

\subsection{Computational details}

All calculations have been processed in Gaussian 03 package. ${ }^{30}$ The magnetic isotropic shielding tensors were carried out with the hybrid DFT method on the basis of B3LYP functional. ${ }^{31}$ The experimentally determined geometries for the complete structures of compounds 1-3 were used for the calculation of the magnetic exchange coupling constants. ${ }^{32}$ Neither variation of the geometrical parameters nor the geometry optimization was attempted in this calculation because a small variation in the geometry can have a big effect on the calculated magnetic interaction parameters.

\subsection{Synthesis of [Co(Htatb)(1,3-bimyb)] (1)}

A mixture of $\mathrm{CoCl}_{2} \cdot 6 \mathrm{H}_{2} \mathrm{O}(0.1 \mathrm{mmol}, 0.024 \mathrm{~g}), \mathrm{H}_{3} \mathrm{tatb}(0.1 \mathrm{mmol}$, $0.026 \mathrm{~g}$ ), 1,3-bimyb (0.1 mmol, $0.024 \mathrm{~g})$ and $8 \mathrm{~mL} \mathrm{DMF-} \mathrm{H}_{2} \mathrm{O}$ $(\mathrm{V}: \mathrm{V}=1: 1)$ was stirred for $30 \mathrm{~min}$ in air. The mixture was then transferred to a $20 \mathrm{ml}$ airtight glass reactor and kept at $100{ }^{\circ} \mathrm{C}$ for 5 days under autogenous pressure, and then cooled to room temperature at a rate of $5{ }^{\circ} \mathrm{C} \mathrm{h}^{-1}$. Red crystals of 1 were obtained and washed with DMF and dried in the air (yield: $53 \%$ based on Co). Elemental analysis (\%). Calcd for $\mathrm{C}_{38} \mathrm{H}_{27} \mathrm{~N}_{7} \mathrm{O}_{6} \mathrm{Co}$ : C, 61.96; H, 3.69; N, 13.31\%. Found: C, 61.64; H, 3.78; N, 13.56\%. IR data (KBr cm $\left.{ }^{-1}\right): 3412(\mathrm{~s}), 2358(\mathrm{w}), 1711(\mathrm{~s}), 1652$ (s), $1547(\mathrm{~m}), 1502$ (m), $1406(\mathrm{~m}), 1134(\mathrm{w}), 1072(\mathrm{w}), 837$ (m), $765(\mathrm{~m}), 672(\mathrm{w})$, $585(\mathrm{w})$.

\subsection{Synthesis of $[\mathrm{Co}($ Htatb)(bimbp) $] \cdot$ DMF (2)}

Complex 2 was prepared as for 1 by using bimbp ligand $(0.1 \mathrm{mmol}, 0.029 \mathrm{~g})$ instead of 1,3-bimyb. Red crystals of 2 were obtained and washed with DMF and dried in the air (yield: 56\% based on Co). Elemental analysis. Calcd for $\mathrm{C}_{45} \mathrm{H}_{34} \mathrm{~N}_{8} \mathrm{O}_{7} \mathrm{Co}$ : C, 63.01; H, 3.99; N, 13.06\%. Found: C, 63.17; H, 3.87; N, 13.27\%. IR data (KBr cm $\left.{ }^{-1}\right): 3429$ (s), 2107 (m), 1714 (s), 1648 (s), 1521 (m), 1454 (s), 1402 (s), 1154 (m), 1018 (w), 872 (m), 765 (m), $669(\mathrm{~m})$.

\subsection{Synthesis of $\left[\mathrm{Co}(\mathrm{Htatb})(1,4\right.$-bimyb) $] \cdot \mathrm{H}_{2} \mathrm{O}$ (3)}

The procedure is similar to that of $\mathbf{1}$, except that the 1,3-bimyb ligand was replaced by 1,3-bimyb ligand ( $0.1 \mathrm{mmol}, 0.024 \mathrm{~g}$ ), and the mixture was heated at $100{ }^{\circ} \mathrm{C}$ for 5 days, and then it was cooled to room temperature at $5{ }^{\circ} \mathrm{C} \mathrm{h}^{-1}$. Purple crystals of 3 were obtained and washed with DMF and dried in the air (yield: $54 \%$ based on Co). Elemental analysis (\%). Calcd for $\mathrm{C}_{38} \mathrm{H}_{29} \mathrm{~N}_{7} \mathrm{O}_{7} \mathrm{Co}$ : C 60.48, $\mathrm{H}$ 3.87, N 12.99; found: $\mathrm{C} 60.75 ; \mathrm{H}$ 3.64; N 12.76; IR data $\left(\mathrm{KBr} \mathrm{cm}^{-1}\right)$ : 3421 (s), $2374(\mathrm{w}), 1718(\mathrm{~s})$, 1654 (s), 1560 (m), 1508 (m), 1396 (m), 1234 (w), 1083 (w), 825 (m), $771(\mathrm{~m}), 653(\mathrm{w}), 572(\mathrm{w})$.

\subsection{X-ray crystallographic studies}

Diffraction intensities for the three complexes were collected at $293 \mathrm{~K}$ on a Bruker SMART $1000 \mathrm{CCD}$ diffractometer employing graphite-monochromated Mo-K $\alpha$ radiation $(\lambda=0.71073 \AA)$. A semi-empirical absorption correction was applied using the SADABS program. ${ }^{33}$ The structures were solved by direct methods and refined by full-matrix least-squares on $F^{2}$ using the SHELXS 2014 and SHELXL 2014 programs, respectively. ${ }^{\mathbf{3 4 , 3 5}}$ Non-hydrogen atoms were refined anisotropically and hydrogen atoms were placed in geometrically calculated positions. The crystallographic data for compounds 1-3 are listed in Table 1, and selected bond lengths and angles are listed in Table S1.†

\section{Results and discussion}

\subsection{Crystal structures of [Co(Htatb)(1,3-bimyb)] (1)}

Single-crystal X-ray analysis reveals that complex 1 shows a double-strand 1D chain structure. The asymmetric unit of 1 consists of one independent $\mathrm{Co}^{\mathrm{II}}$ ion, one Htatb ligand, one 1,3-bimyb ligand. Each $\mathrm{Co}^{\mathrm{II}}$ ion coordinates to two nitrogen atoms from two 1,3-bimyb ligand, four carboxylate oxygen atoms from three Htatb ligands, forming a $\mathrm{CoN}_{2} \mathrm{O}_{4}$ distorted octahedral geometry (see Fig. 1a). The bond lengths of $\mathrm{Co}-\mathrm{O} /$ $\mathrm{N}$ are in the range of 2.0104(18) A to 2.0045(17) $\mathrm{A}$, the $\mathrm{O} / \mathrm{N}-\mathrm{Co}-$ $\mathrm{O} / \mathrm{N}$ bond angles cover the range of $59.41(6)-177.70(8)^{\circ}$. In compound $\mathbf{1}$, the tripodal carboxylate ligands are partly deprotonated and one carboxylate group adopts $\mu_{2}-\eta^{1}-\eta^{1}$ bridging mode to link two $\mathrm{Co}^{\mathrm{II}}$ ions, and another one adopts $\mu_{1}-\eta^{1}-\eta^{1}$ chelating mode to link one $\mathrm{Co}^{\mathrm{II}}$ ion, resulting in a double-strand chain structure (Fig. 1b). The flexible 1,3bimyb ligand joins the adjoining two $\mathrm{Co}^{\mathrm{II}}$ ions to form a ring on both sides of a $1 \mathrm{D}$ chain, with the Co $\cdots$ Co separation is $4.176 \AA$ A. The adjacent chains are joined to generate a 2D layer 
Table 1 Crystal data and structural refinement summary of compounds 1-3

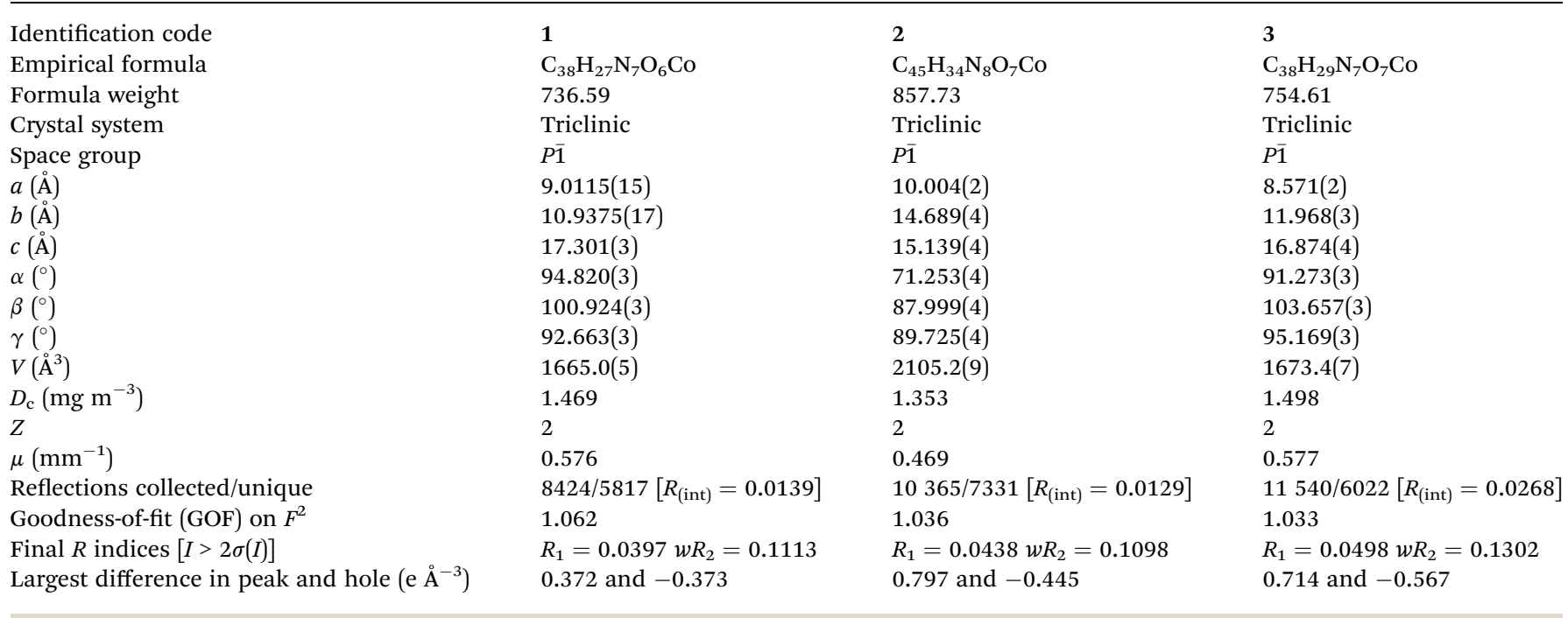

structure through intermolecular $\mathrm{O}-\mathrm{H} \cdots \mathrm{O}$ hydrogen bonds [O3-H3A $\cdots$ O4 distance: 2.6478(18) $\AA$, angle: 176.95(4)] (Fig. 1c). Further, through aromatic $\pi-\pi$ stacking interactions between phenyl rings of two Htatb ligands (centroid-tocentroid distance: 3.681(3) $\AA$ ), the adjacent layers generate a 3D supramolecular structure (Fig. S1 in the ESI $\dagger$ ).

\subsection{Crystal structures of [Co(Htatb)(bimbp)] DMF (2)}

Compound 2 shows a 2D structure with 1D channel, and compound 2 is made up of the $\mathrm{Co}^{\mathrm{II}}$ ion, Htatb ligand, bimbp ligand and free DMF molecule. Each $\mathrm{Co}^{\mathrm{II}}$ ion is located in a distorted octahedral geometry and is coordinated to four oxygen atoms of two Htatb ligand and two nitrogen atoms of two bimbp ligands, as shown in Fig. 2a. The bond lengths of $\mathrm{Co}-\mathrm{O} / \mathrm{N}$ are in the range of 2.0394(19)-2.2578(19) $\AA$, the $\mathrm{O} / \mathrm{N}-$ $\mathrm{Co}-\mathrm{O} / \mathrm{N}$ bond angles cover the range of 59.20(7)-178.24(8) ${ }^{\circ}$. As compound 1 is same, the carboxylic groups of Htatb ligand have two coordination modes: one carboxylate group adopts $\mu_{2}-\eta^{1}-\eta^{1}$ bridging mode to link two $\mathrm{Co}^{\mathrm{II}}$ ions, and another one adopts $\mu_{1}-\eta^{1}-\eta^{1}$ chelating mode to link one $\mathrm{Co}^{\mathrm{II}}$ ion, resulting in a double-strand chain structure (Fig. S2 in the ESI + ). Through bimbp ligands bridging, the adjacent chains are connected to generate a 2D structure with 1D channel (See Fig. $2 \mathrm{~b}$ and $\mathrm{S} 3 \dagger$ ). The potential volume accessible for DMF, determined by PLATON calculation, is $410.3 \AA^{3}$ per unit cell volume $\left(2105.2 \AA^{3}\right)$, which represents $19.5 \%$ void per unit volume for 2 .

\subsection{Crystal structures of $[\mathrm{Co}(\mathrm{Htatb})(1,4-b i m y b)] \cdot \mathrm{H}_{2} \mathrm{O}$ (3)}

Complex 3 exhibits a 4 -fold interpenetrating 3D structure. The asymmetric unit of 3 comprises one $\mathrm{Co}^{\mathrm{II}}$ ion, one Htatb ligand, one 1,4-bimyb ligand and one free water molecule, which is disposed about a centre of inversion. Each fourcoordinated $\mathrm{Co}^{\mathrm{II}}$ center is surrounded by two nitrogen atoms coming from two 1,4-bimyb ligands, and two oxygen atoms from two Htatb ligands, taking a distorted tetrahedral geometry (Fig. 3a). The bond lengths of $\mathrm{Co}-\mathrm{O}$ and $\mathrm{Co}-\mathrm{N}$ are in the range of 1.973(2)-2.040(3) A. Compared with complexes 1 and 2 , the carboxylic groups of 3 adopts $\mu_{1}-\eta^{1}-\eta^{0}$ and $\mu_{1}-\eta^{1}-$ $\eta^{0}$ bridging mode to link two $\mathrm{Co}^{\mathrm{II}}$ ions, resulting in a $1 \mathrm{D}$ chain structure, through 1,4-bimyb ligand bridging, the adjacent chains are connected to generate a 2D layer structure (Fig. S4†). These 2D layers are further joined by $\mathrm{O}-\mathrm{H} \cdots \mathrm{O}$

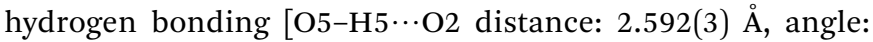
176.95(4)] to produce a 3D architecture (Fig. 3b). In order to simplify the 3D framework, we considered the Htatb anion as a 3-connected node and $\mathrm{Co}^{\mathrm{II}}$ ion as a 5 -connected node, 1,4bimyb ligand as linkers, the whole framework can be simplified as a 3,5-connected 2-nodal net topology with a point symbol of $\left(3 \cdot 7^{2}\right)\left(3^{2} \cdot 7^{5} \cdot 8^{3}\right)$ (Fig. 3c). However, due to the absence of large guest molecules to fill the void space, the potential voids are filled via mutual interpenetration of three independent equivalent frameworks, generating a four-fold interpenetrating 3D architecture (Fig. 3d).

\subsection{Structural diversity of 1-3}

It is noteworthy that a variety of framework structures can be achieved on the basis of the choice of $\mathrm{H}_{3}$ tatb and imidazolecontaining ligands. From Htatb containing compounds 1-3, Htatb ligand shows two types of coordination modes: one adopts $\mu_{2}-\eta^{1}-\eta^{1}$ and $\mu_{1}-\eta^{1}-\eta^{1}$ bridging mode for $\mathbf{1}$ and 2 , another adopts $\mu_{1}-\eta^{1}-\eta^{0}$ and $\mu_{1}-\eta^{1}-\eta^{0}$ bridging mode for $\mathbf{3}$, and then the different $\mathrm{N}$-containing co-ligands were employed, resulting in three different dimensional frameworks (compound 1 for $1 \mathrm{D}$, compound $\mathbf{2}$ for 2D, compound 3 from $2 \mathrm{D}$ to $3 \mathrm{D}$ ). The different imidazole-containing ligands may affect the structure of compounds, when a flexible ligand (1,3-bimyb) is employed to generate 1D structure for $\mathbf{1}$, when a rigid ligand (bimbp) is attended, the 2D structure of 2 are obtained, when a semi-rigid ligand is used, a 4 -fold interpenetrating 3D architecture for 3 is obtained. By 
(a)

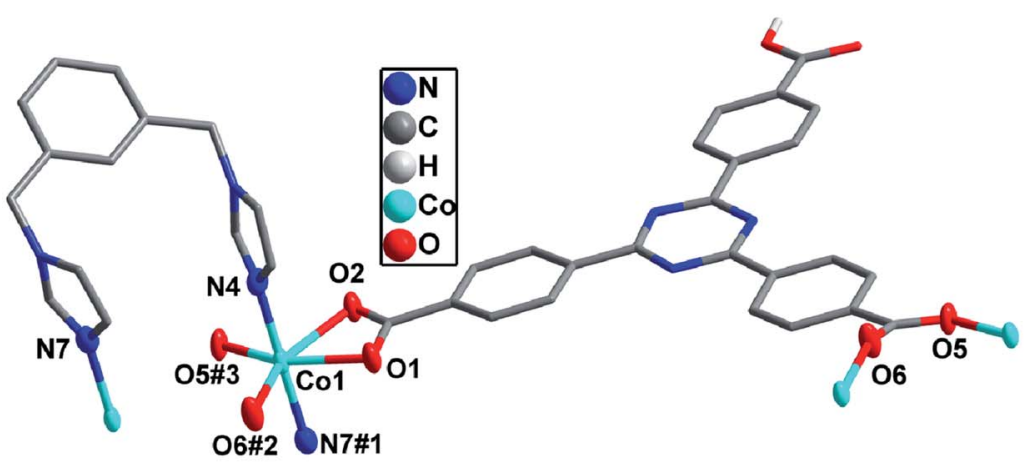

(b)

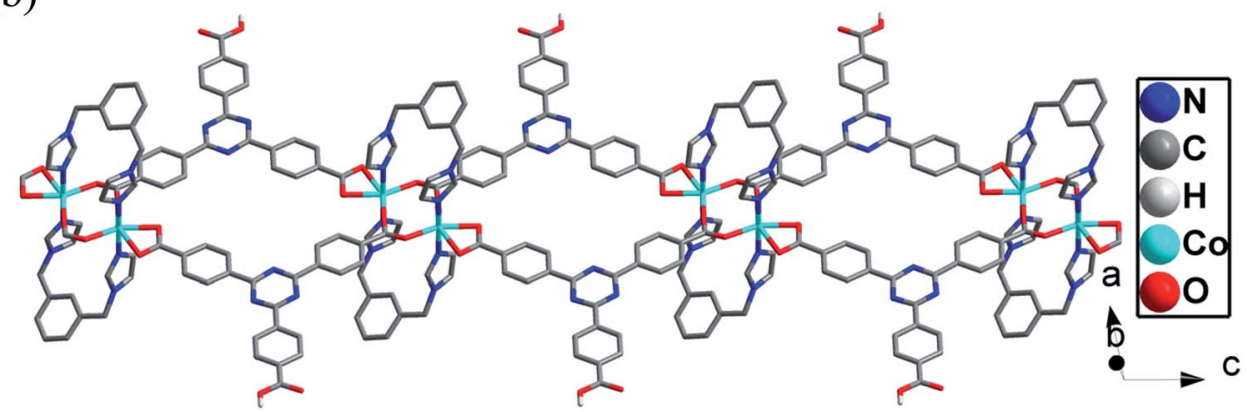

(c)

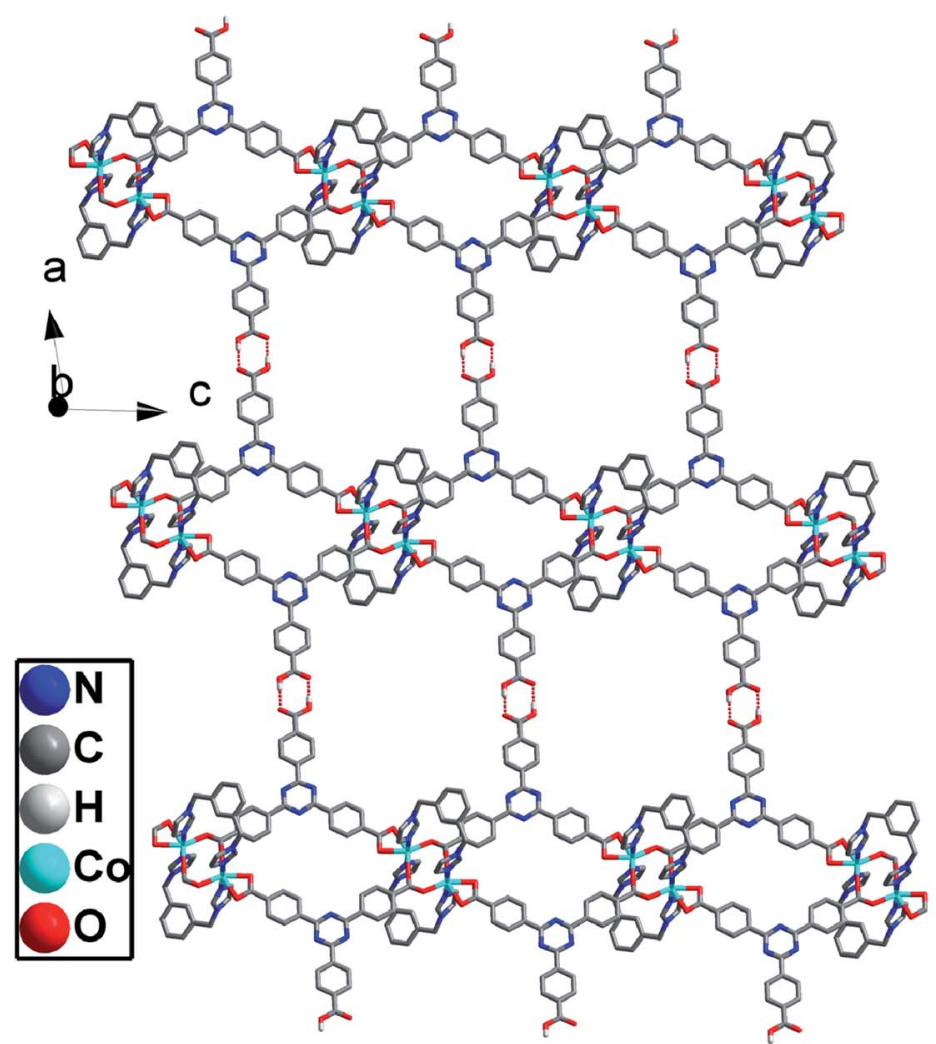

Fig. 1 (a) The coordination environments of $\mathrm{Co}(I)$ ion in compound 1. Hydrogen atoms are omitted for clarity except the Htatb ligand. Displacement ellipsoids are drawn at the $50 \%$ probability level (symmetry code: $\# 1-x+1,-y+1,-z+1, \# 2 x, y, z-1, \# 3-x+1,-y+1,-z)(b)$ The double-strand chain structure of 1 . (c) The 2D layer structure of 1. 
(a)

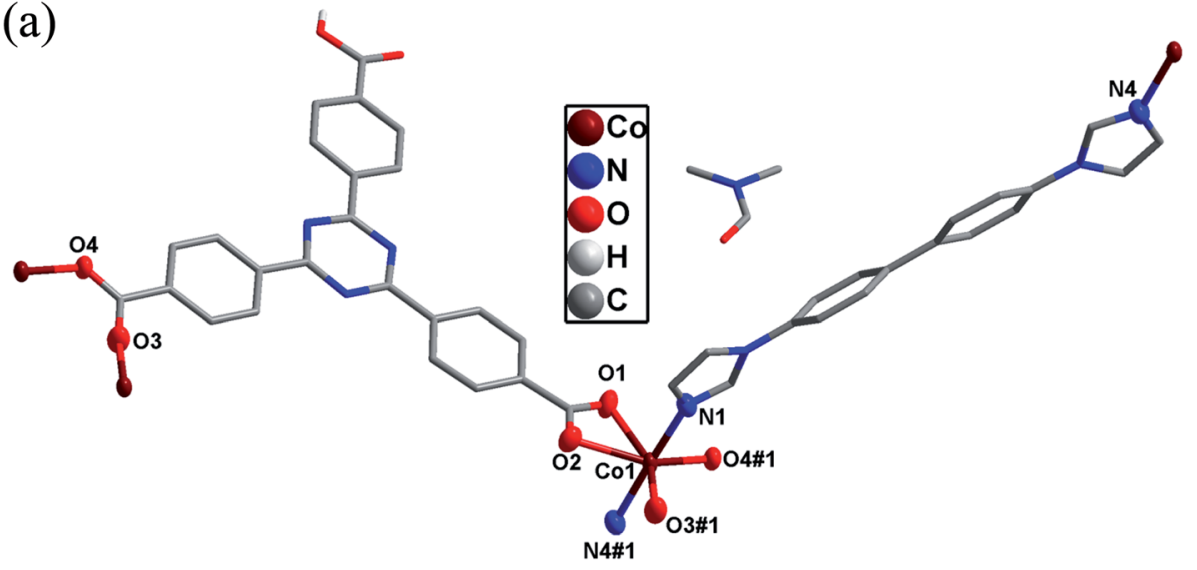

(b)

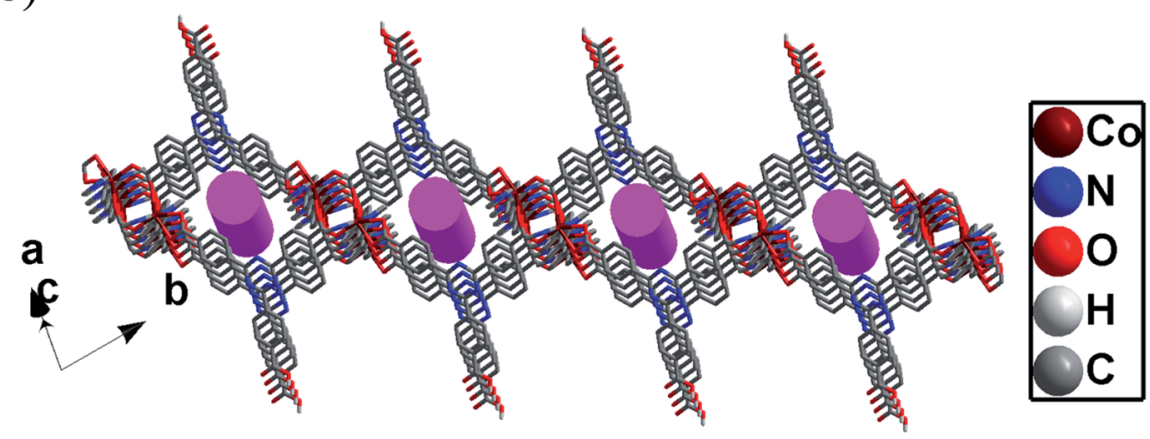

Fig. 2 (a) The coordination environments of Co(I) ion in 2. Hydrogen atoms are omitted for clarity except the Htatb ligand. (symmetry code: \#1 $-x-1,-y+1,-z+2, \# 2 x, y+1, z-1, \# 3 x-1, y, z+1)$. (b) The 2D structure with 1D channel of 2.

(a)

(c)

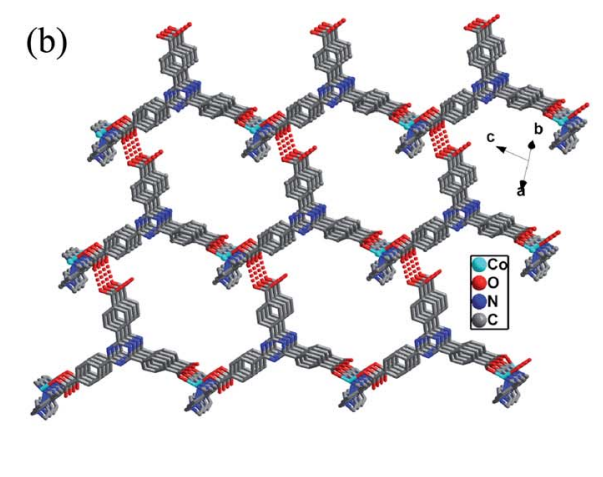

(d)

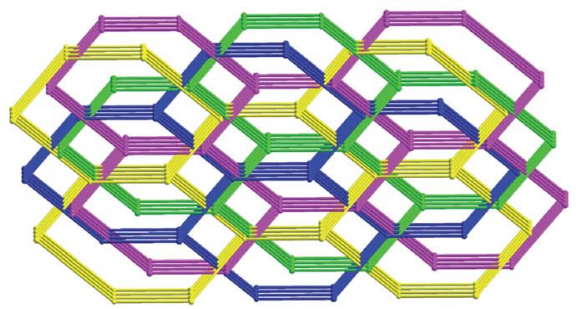

Fig. 3 (a) The coordination environments of Co(II) ion in 3. Hydrogen atoms are omitted for clarity except the Htatb ligand. (symmetry code: \#1 $x$ $+1, y, z+1, \# 2 x-1, y-1, z$ ). (b) The 3D architecture of 3. (c) The 3,5-connected net topology of 3. (d) The four-fold interpenetrating 3D architecture of 3 . 
comparing of the structures of compounds $1-3$, the $\mathrm{H}_{3}$ tatb and imidazole-containing ligands may tune the final structural features.

\subsection{Thermogravimetric analysis}

To study the thermal stability of 1-3, thermogravimetric (TG) analyses were performed on polycrystalline samples under a nitrogen atmosphere with a heating rate of $10{ }^{\circ} \mathrm{C} \min ^{-1}$ (see Fig. S5-S7†). The TG curve of 1 reveals that no weight loss occurs from $20^{\circ} \mathrm{C}$ until about $220^{\circ} \mathrm{C}$, above which, a significant weight loss of $92.4 \%$ is observed and ended at about $460{ }^{\circ} \mathrm{C}$ indicating the release the organic ligands (calcd. 92.0\%). The TG curve of 2 shows two step weight losses, the first weight loss in the range 50-140 ${ }^{\circ} \mathrm{C}$ (obsd $8.7 \%$, calcd. 8.5\%) was assignable to the loss of DMF molecules. The second weight loss of $85.3 \%$ in the temperature range 210 to $470{ }^{\circ} \mathrm{C}$ corresponds to the release of the Htatb and bimbp ligands (calcd. 84.6\%). TG curve of 3 reveals that the first weight loss of $2.6 \%$ from $40{ }^{\circ} \mathrm{C}$ to $120{ }^{\circ} \mathrm{C}$ corresponds to the loss of the lattice water molecules (calcd. $2.4 \%$ ), and then the larger weight loss(obsd 90.4\%) occurred in the range of $230-500{ }^{\circ} \mathrm{C}$, corresponding to the decomposition of the Htatb and 1,4-bimyb ligands (calcd. 89.8\%). The final decomposition products of 1-3 were confirmed to be $\mathrm{CoO}$, which have also been further confirmed by PXRD patterns of compounds.

\subsection{Infrared spectra of complexes 1-3}

IR spectra of complexes 1-3 show features attributable to compositions of the coordination polymers. The observed strong characteristic peaks appearing around 3412, 3429 and $3421 \mathrm{~cm}^{-1}$ in spectra are attributed to the $\mathrm{O}-\mathrm{H}$ stretching vibrations, respectively. ${ }^{36}$ Due to partial deprotonation of carboxylate in complexes 1-3, the absorptions about 1711, 1714 and $1718 \mathrm{~cm}^{-1}$ can be attributed to the stretching vibrations of the $v_{\mathrm{COOH}}$ in the carboxylate. ${ }^{37,38}$ The presence of the characteristic bands at 1652, 1648 and $1654 \mathrm{~cm}^{-1}$ for $1-3$

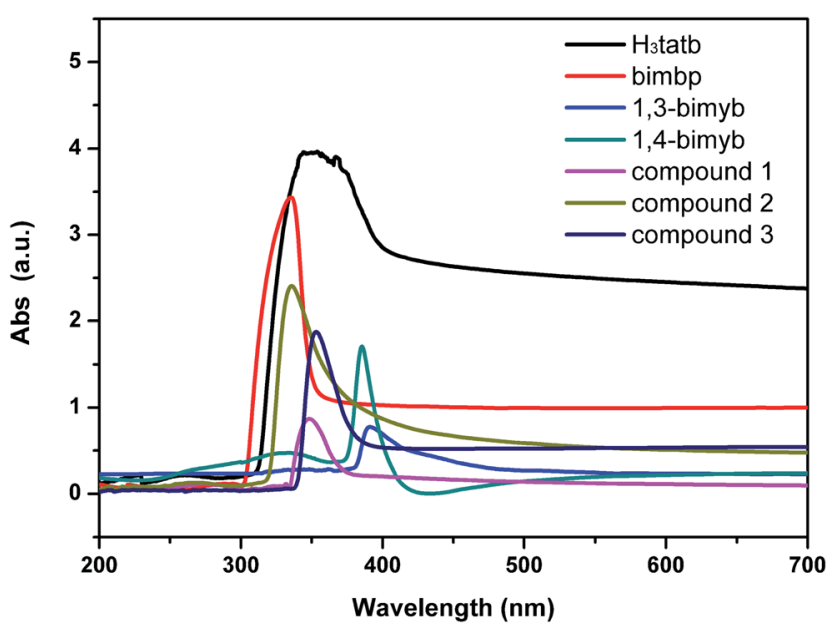

Fig. 4 The UV-vis spectrum of the free ligands and complexes 1-3 in DMF- $\mathrm{H}_{2} \mathrm{O}(\mathrm{V}: \mathrm{V}=1: 1)$. suggest the $v_{-\mathrm{C}=\mathrm{N}-}$ stretching vibrations of Htatb ligand. The intense characteristic peaks appearing around 1547 and 1502, $1406 \mathrm{~cm}^{-1}$ for 1,1521 and $1454,1348 \mathrm{~cm}^{-1}$ for 2,1560 and $1508,1396 \mathrm{~cm}^{-1}$ for 3 in the IR spectra correspond to asymmetric and symmetric stretching vibrations of carboxylic groups, respectively. ${ }^{39,40}$ The presence of the characteristic bands at 1134,1154 and $1234 \mathrm{~cm}^{-1}$ for 1-3 suggest the $v_{\mathrm{C}-\mathrm{O}}$ stretching vibrations. The presence of the characteristic bands at $1072 \mathrm{~cm}^{-1}$ for $1,1018 \mathrm{~cm}^{-1}$ for $2,1083 \mathrm{~cm}^{-1}$ for 3 suggest the $v_{\mathrm{C}-\mathrm{N}}$ stretching vibrations of the imidazole ring. ${ }^{41}$ The presence of the characteristic bands at $1072 \mathrm{~cm}^{-1}$ for $\mathbf{1}$, $1018 \mathrm{~cm}^{-1}$ for $2,1083 \mathrm{~cm}^{-1}$ for 3 suggest the $v_{\mathrm{C}-\mathrm{N}}$ stretching vibrations of the imidazole ring. ${ }^{42}$ The absorptions about $650-$ $880 \mathrm{~cm}^{-1}$ of 1-3 can be attributed to the $v_{\mathrm{C}-\mathrm{H}}$ bending vibrations of phenyl ring.

\subsection{UV-vis spectroscopy}

The UV-vis spectrum of the free ligands and complexes 1-3 in DMF- $\mathrm{H}_{2} \mathrm{O}(\mathrm{V}: \mathrm{V}=1: 1)$ is described in Fig. 4. In the spectra of the $\mathrm{H}_{3}$ tatb ligand, one broad and strong absorption band at $354 \mathrm{~nm}$ occurred in the range of 300-400 $\mathrm{nm}$, and the absorption bands of the bimbp, 1,3-bimyb and 1,4-bimyb ligands, found at $335 \mathrm{~nm}, 391 \mathrm{~nm}$ and $386 \mathrm{~nm}$, can be assigned to the $\pi \rightarrow \pi^{*}$ and $\mathrm{n} \rightarrow \pi^{*}$ transitions of the these ligands. In the spectra of complexes 1 and 3, the maximum of absorbance are observed at $348 \mathrm{~nm}$ (1) and $351 \mathrm{~nm}$ (3), can be assigned to the $\mathrm{n} \rightarrow \pi^{*}$ transitions of the ligands and exhibit slight blue shifted compared to the $\mathrm{H}_{3}$ tatb ligand (354 nm), these shifts reflect coordination of the ligands to the $\mathrm{Co}$ (II) atom. The absorption bands of complex 2 was observed at $336 \mathrm{~nm}(2)$, which can be attributed to the intraligand $\pi \rightarrow \pi^{*}$ and $n \rightarrow \pi^{*}$ transitions of the coordinated ligands.

\subsection{Photoluminescence properties}

The luminescent emission spectra of 1-3 were examined in the solid state at room temperature as is shown in Fig. 5. The main

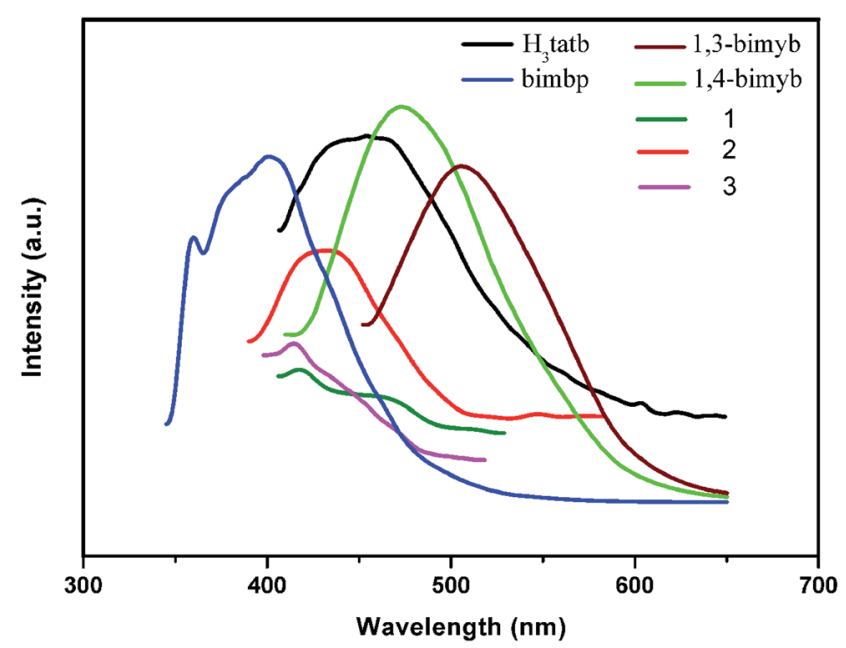

Fig. 5 The solid state luminescent emissions of complexes 1-3 and ligands. 
emission peak of the free ligand $\mathrm{H}_{3}$ tatb appears at $454 \mathrm{~nm}\left(\lambda_{\text {ex }}\right.$ $=364 \mathrm{~nm}$ ) and can be assigned to the intra-ligand $\pi^{*}-\pi$ transitions. ${ }^{\mathbf{4}}$ Complexes $\mathbf{1}$ and $\mathbf{3}$ show similar weaker emission peaks at $414 \mathrm{~nm}$ and $417 \mathrm{~nm}\left(\lambda_{\mathrm{ex}}=366 \mathrm{~nm}\right)$, however, the intense emission of 1,3-bimyb and 1,4-bimyb ligand were observed at $506 \mathrm{~nm}\left(\lambda_{\mathrm{ex}}=433 \mathrm{~nm}\right)$ and $473 \mathrm{~nm}\left(\lambda_{\mathrm{ex}}=400 \mathrm{~nm}\right)$, respectively. Relative to their ligands, complexes 1 and 3 show a blue shift, probably owing to ligand to metal charge transfer (LMCT) ${ }^{44,45}$ Complex 2 show an emission peaks at $432 \mathrm{~nm}\left(\lambda_{\text {ex }}\right.$ $=345 \mathrm{~nm}$ ), in comparison with that of free $\mathrm{H}_{3}$ tatb and bimbp ligand (an intense emission at $401 \mathrm{~nm}\left(\lambda_{\text {ex }}=340 \mathrm{~nm}\right)$ ), which are attributed to $\mathrm{H}_{3}$ tatb or bimbp ligand-based charge transfer. ${ }^{46,47}$

\subsection{Magnetic property}

The magnetic susceptibilities $\left(\chi_{M}\right)$ of $\mathbf{1}$ and $\mathbf{2}$ were measured in the temperature range of $2-300 \mathrm{~K}$ with a field of $1000 \mathrm{Oe}$. To simulate the experimental magnetic behavior, we analyzed the experimental $\chi_{M} T$ data of 1 and 2 by the dinuclear models as expressed by eqn (1). ${ }^{48}$

$$
\begin{aligned}
\chi_{\mathrm{M}} & =\frac{2 N g^{2} \beta^{2}}{k T} \\
& \times \frac{\exp (-2 J / k T)+5 \exp (-6 J / k T)+14 \exp (-12 J / k T)}{1+3 \exp (-2 J / k T)+5 \exp (-6 J / k T)+7 \exp (-12 J / k T)}
\end{aligned}
$$

$N, g, \beta, k$ and $T$ are the Avogadro number, Zeeman factor, Bohr magneton, Boltzmann constant and temperature in kelvin, respectively. $J$ is the exchange coupling constant between adjacent $\mathrm{Co}(\mathrm{II})$ ions.

The magnetic results of complex 1 are illustrated in Fig. 6a. As observed, the experimental $\chi_{\mathrm{M}} T$ value at $300 \mathrm{~K}$ is $3.796 \mathrm{~cm}^{3} \cdot \mathrm{K}$ $\mathrm{mol}^{-1}$, which is somewhat larger than the expected value 3.75 $\left.\mathrm{cm}^{3} \cdot \mathrm{K} \mathrm{mol}^{-1}\right)$ of the two isolated spin-only Co(II) ions $(S=3 / 2)$. The distance between two closest Co(II) centers aligned in the $1 \mathrm{D}$ chain is $4.176 \AA$. When the temperature decreases, the $\chi_{\mathrm{M}} T$ descends gradually until $75 \mathrm{~K}$ and then rapidly drops to $2 \mathrm{~K}$. The temperature dependence of the reciprocal susceptibility $\left(1 / \chi_{M}\right)$ obeys the Curie-Weiss law above $30 \mathrm{~K}$ with $\theta=-8.44 \mathrm{~K}, C=3.69$ $\mathrm{cm}^{3} \mathrm{~K} \mathrm{~mol}^{-1}$ and $R=6.24 \times 10^{-4}\left(R=\sum\left[\left(\chi_{\mathrm{M}}\right)_{\mathrm{obs}}-\left(\chi_{\mathrm{M}}\right)_{\mathrm{calc}}\right]^{2} /\right.$ $\left.\sum\left[\left(\chi_{\mathrm{M}}\right)_{\mathrm{obs}}\right]^{2}\right)$. The best fitting for the experimental data was found with $J=-7.27 \mathrm{~cm}^{-1}, g=2.24$ and $R=4.13 \times 10^{-4}$. The values of $J$ and $\theta$ indicate the antiferromagnetic interactions between adjacent $\mathrm{Co}(\mathrm{II})(S=3 / 2)$ ions.

The experimental $\chi_{\mathrm{M}} T$ values of 2 is $3.713 \mathrm{~cm}^{3} \mathrm{~K} \mathrm{~mol}^{-1}$ at room temperature (Fig. 6b), which are very close to the expected value of $3.75 \mathrm{~cm}^{3} \mathrm{~K} \mathrm{~mol}^{-1}$ for two isolated spin-only Co(II) ions with $S=3 / 2$. As the temperature decreases, the $\chi_{\mathrm{M}} T$ value gradually decreases till $50 \mathrm{~K}$ to reach a value of $2.73 \mathrm{~cm}^{3} \mathrm{~K} \mathrm{~mol}^{-1}$ after which an obvious fall was appeared to reach a value of $0.03 \mathrm{~cm}^{3} \mathrm{~K} \mathrm{~mol}^{-1}$ at $2 \mathrm{~K}$, manifesting a significant antiferromagnetic exchange between the magnetic centres in $\mathrm{Co}_{2}$ dimer. The temperature dependence of the reciprocal susceptibility $\left(1 / \chi_{\mathrm{M}}\right)$ obeys the Curie-Weiss law above $30 \mathrm{~K}$ with $\theta=-7.21 \mathrm{~K}, C=3.73 \mathrm{~cm}^{3} \mathrm{~K} \mathrm{~mol}^{-1}$ and $R=5.17 \times 10^{-4}$. The best-fit parameters for the experimental data gives, $J=-9.42 \mathrm{~cm}^{-1}, g=2.28$ and $R=1.14 \times 10^{-4}$. In 2, the magnetic coupling between two $\mathrm{Co}(\mathrm{II})$ centers is transmitted through two carboxylate bridges, the Co $\cdots$ Co distance of $4.156 \AA$ is responsible for the antiferromagnetic coupling. In these compounds, this behavior indicates a dominant antiferromagnetic interaction between the $\operatorname{Co}(\mathrm{II})$ ions for 1 and 2. However, complex 3 features a 3D supramolecular structure, the Co $\cdots$ Co distances through the Htatb and 1,4bimyb ligands bridging are 17.026 and $14.079 \AA$, due to the large distance between adjacent $\mathrm{Co}(\mathrm{II})$ ions, we did not discuss the magnetic behavior of 3 .

\subsection{Magnetic properties of DFT calculations}

The DFT calculations have been widely proved to be one of the most efficient tools to investigate magnetic structure of transition metal complexes. ${ }^{49-51}$ We used a phenomenological Heisenberg Hamiltonian $\hat{H}=-J \hat{S}_{1} \hat{S}_{2}$ to describe the exchange coupling in a dinuclear compound, the coupling constant $J$ can be related to the energy difference between the lowest and highest spin states. For the case in which $S_{1}=S_{2}$, the coupling constant may be obtained by using the following eqn (3). ${ }^{52}$

$$
E_{\mathrm{HS}}-E_{\mathrm{LS}}=-2 J S_{\mathrm{i}}\left(S_{\mathrm{i}}+1 / 2\right)
$$

where $E_{\mathrm{HS}}$ is the energy that corresponds to the state with the highest total spin, $E_{\mathrm{LS}}$ corresponds to the state with the lowest total spin $(S=0)$, and $S_{\mathrm{i}}$ is the total spin on each metal atom.

When using DFT-based wave functions, a reasonable estimate of the energy corresponding to the low spin state, $E_{\mathrm{LS}}$ can be obtained directly from the energy of a broken-symmetry solution $E_{\mathrm{BS}}$. In this case, compounds 1 and 2 are 1D chain and 2D layer, and the theoretical calculations would be a difficult task for such a large periodical system. Here, the monomer $\left[\mathrm{Co}_{2}(\text { Htatb })_{2}(1,3 \text {-bimyb })_{2}\right]$ of $1\left[\mathrm{Co}_{2}(\mathrm{Htatb})_{2}(\mathrm{bim}\right.$ $\left.\mathrm{bp})_{2}\right]$ of 2 were intercepted, we arrive to the following expressions for $J$ :

$$
E_{\mathrm{HS}}-E_{\mathrm{BS}}=-6 J_{\mathrm{Co}-\mathrm{Co}}
$$

The calculated coupling constant $J$ and related quantities are listed in Table 2. We have used via eqn (3) to estimate the magnetic coupling constants $(J)$ of compounds 1 and 2 . The computed $J$ values $\left(J=-9.04 \mathrm{~cm}^{-1}\right.$ for 1 and $J=-11.63 \mathrm{~cm}^{-1}$ for 2) predict antiferromagnetic, the results were agreed with the experimental data. We have used the non-spin projected, giving a better agreement for compounds 1 and 2. This is due to the strong localization of the wavefunction at the metal centers both computational techniques produce results that are in remarkable agreement with the experimental value. Full molecule calculation using hybrid B3LYP functional to reproduce BS-HS energy gap of compounds is not only found to be successful in describing the magnetic behavior of the compounds correctly in this study but also yielded $J$ that are in excellent agreement with the experimental value. So the full molecule DFT based calculation based on X-ray structural data could be successfully used to rationalize the magnetic behavior of this class of compounds. 

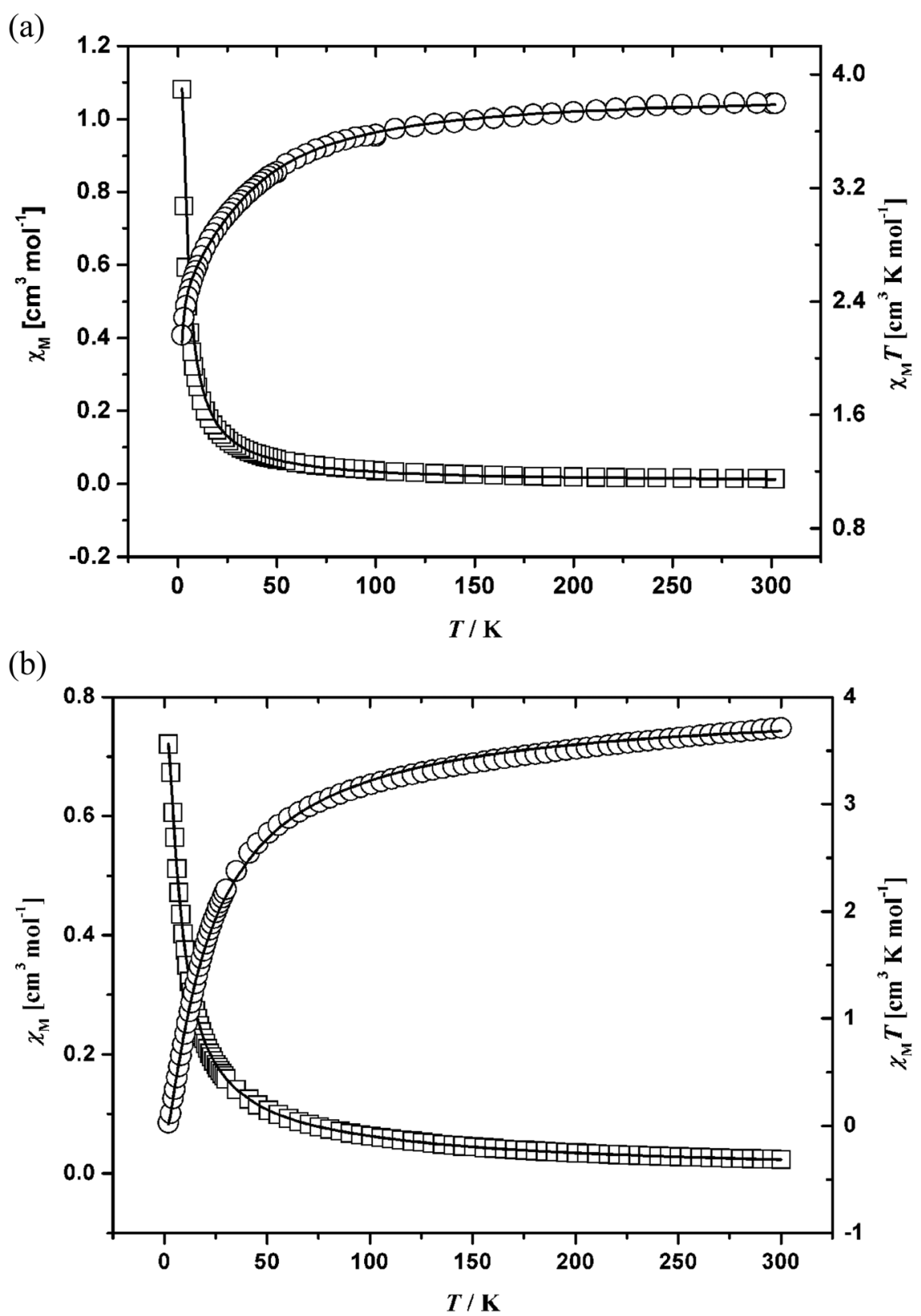

Fig. 6 Thermal variation of $\chi_{M}$ and $\chi_{M} T$ for $1(a)$ and 2 (b) $\left(O, \chi_{M}\right.$ experimental values, $\square, \chi_{M} T$ experimental values and the solid line represents the best fit obtained from the Hamiltonian given in the text.).

Table 2 Calculated energy (au.) and magnetic exchange constant $\mathrm{J}$ $\left(\mathrm{cm}^{-1}\right)$ for 1 and 2

\begin{tabular}{lcrrr}
\hline & & & \multicolumn{2}{c}{ Calculation, Experiment, } \\
Compound & $E_{\mathrm{BS}} /($ au. $)$ & $E_{\mathrm{HS}} /($ au. $)$ & $J /\left(\mathrm{cm}^{-1}\right)$ & $J /\left(\mathrm{cm}^{-1}\right)$ \\
\hline 1 & -9684.132164 & -9684.131917 & -9.04 & -7.27 \\
2 & -10160.989084 & -10160.988766 & -11.63 & -9.42
\end{tabular}

\section{Conclusions}

In summary, three cobalt-based coordination polymers have been synthesized by the self-assembly of $\mathrm{Co}(\mathrm{II})$ salts with $\mathrm{H}_{3}$ tatb and imidazole-containing Ligands. Assemblies of these compounds generate three types of diverse frameworks: one 1D chain, one 2D layer and 2D to 3D structure. By comparing of the structures of 1-3, the N-donor ligand can also slightly tune the final structural features. Moreover, the fluorescent properties of 1-3 and magnetic behavior of $\mathbf{1}$ and $\mathbf{2}$ have also been investigated. According to the crystal structures, the DFT-BS approach was applied to study the magnetic coupling behavior for $\mathbf{1}$ and 2, the result reveals that the calculated exchange coupling constants $J$ were in good agreement with the experimental data.

\section{Conflicts of interest}

There are no conflicts to declare. 


\section{Acknowledgements}

This work was supported by the National Natural Science Foundation of China (No. 21503183, 21573189, 21663031 and 21763028). The authors thank Professor Wen-Liang Wang (Shaanxi Normal University) for his help.

\section{References}

1 Y. P. Wu, W. Zhou, J. Zhao, W. W. Dong, Y. Q. Lan, D. S. Li, C. H. Sun and X. H. Bu, Angew. Chem., Int. Ed., 2017, 56, 13001-13005.

2 Q. G. Zhai, X. H. Bu, X. Zhao, D. S. Li and P. Y. Feng, Acc. Chem. Res., 2017, 50, 407-417.

3 Y. Zhao, X. G. Yang, X. M. Lu, C. D. Yang, N. N. Fan, Z. T. Yang, L. Y. Wang and L. F. Ma, Inorg. Chem., 2019, 58, 6215-6221.

4 G. Wang, Q. Sun, Y. Liu, B. Huang, Y. Dai, X. Zhang and X. Qin, Chem.-Eur. J., 2015, 21, 2364-2367.

5 Y. L. Wang, J. H. Fu, J. J. Wei, X. Xu, X. F. Li and Q. Y. Liu, Cryst. Growth Des., 2012, 12, 4663-4668.

6 D. S. Li, J. Zhao, Y. P. Wu, B. Liu, L. Bai, K. Zou and M. Du, Inorg. Chem., 2013, 52, 8091-8098.

7 Y. Zhao, L. Wang, N. N. Fan, M. L. Han, G. P. Yang and L. F. Ma, Cryst. Growth Des., 2018, 18, 7114-7121.

8 H. R. Fu, N. Wang, J. H. Qin, M. L. Han, L. F. Ma and F. Wang, Chem. Commun., 2018, 54, 11645-11648.

9 X. Y. Zhang, B. Li and J. P. Zhang, Inorg. Chem., 2016, 55, 3378-3383.

10 I. H. Park, R. Medishetty, J. Y. Kim, S. S. Lee and J. J. Vittal, Angew. Chem., Int. Ed., 2014, 53, 5591-5595.

11 S. Q. Chen, Q. G. Zhai, S. N. Li, Y. C. Jiang and M. C. Hu, Inorg. Chem., 2015, 54, 10-12.

12 Y. He, W. Zhou, G. Qian and B. Chen, Chem. Soc. Rev., 2014, 43, 5657-5658.

13 M. G. Campbell, D. Sheberla, S. F. Liu, T. M. Swager and M. Dinca, Angew. Chem., Int. Ed., 2015, 54, 4349-4352.

14 K. Manna, T. Zhang, F. X. Greene and W. Lin, J. Am. Chem. Soc., 2015, 137, 2665-2673.

15 L. L. Shen, L. Yang, Y. Fan and L. Wang, CrystEngComm, 2015, 17, 9363-9369.

16 W. Lin, Y. Cui, Y. Yu, Q. Hu and G. Qian, Dalton Trans., 2018, 47, 15882-15887.

17 Y. J. Cheng, R. Wang, S. Wang, X. J. Xi, L. F. Ma and S. Q. Zang, Chem. Commun., 2018, 54, 13563-13566.

18 Z. J. Lin, J. Lv, M. C. Hong and R. Cao, Chem. Soc. Rev., 2014, 43, 5867-5895.

19 Y. W. Li, H. Ma, Y. Q. Chen, K. H. He, Z. X. Li and X. H. Bu, Cryst. Growth Des., 2012, 12, 189-196.

20 J. P. Zhang, Y. B. Zhang, J. B. Lin and X. M. Chen, Chem. Rev., 2012, 112, 1001-1033.

21 M. P. Suh, H. J. Park, T. K. Prasad and D. W. Lim, Chem. Rev., 2012, 112, 782-835.

22 S. Q. Ma, D. F. Sun, M. Ambrogio, J. A. Fillinger, S. Parkin and H. C. Zhou, J. Am. Chem. Soc., 2007, 129, 1858-1859.

23 S. Q. Ma, X. S. Wang, D. Q. Yuan and H. C. Zhou, Angew. Chem., Int. Ed., 2008, 47, 4130-4133.
24 W. Gao, F. F. Xing, D. Zhou, M. Shao and S. R. Zhu, Inorg. Chem. Commun., 2011, 14, 601-605.

25 H. B. Zhang, N. Li, C. B. Tian, T. F. Liu, F. L. Du, P. Lin, Z. H. Li and S. W. Du, Cryst. Growth Des., 2012, 12, 670-678.

26 J. W. Rong, W. W. Zhang and J. F. Bai, CrystEngComm, 2016, 18, 7728-7736.

27 C. S. Liu, Z. H. Zhang, M. Chen, H. Zhao, F. H. Duan, D. M. Chen, M. H. Wang, S. Zhang and M. Du, Chem. Commun., 2017, 53, 3941-3944.

28 X. Duan, R. Lv, Z. G. Ji, B. Li, Y. J. Cui, Y. Yang and G. D. Qian, Inorg. Chem. Front., 2018, 5, 1193-1198.

29 X. B. Liu, Z. Y. Xiao, A. Huang, W. Wang, L. L. Zhang, R. M. Wang and D. F. Sun, Z. Anorg. Allg. Chem., 2016, 642, 31-35.

30 M. J. Frisch, G. W. Trucks, H. B. Schlegel, G. E. Scuseria, M. A. Robb, J. R. Cheeseman, Jr, J. A. Montgomery, T. Vreven, K. N. Kudin, J. C. Burant, J. M. Millam, S. S. Iyengar, J. Tomasi, V. Barone, B. Mennucci, M. Cossi, G. Scalmani, N. Rega, G. A. Petersson, H. Nakatsuji, M. Hada, M. Ehara, K. Toyota, R. Fukuda, J. Hasegawa, M. Ishida, T. Nakajima, Y. Honda, O. Kitao, H. Nakai, M. Klene, X. Li, J. E. Knox, H. P. Hratchian, J. B. Cross, V. Bakken, C. Adamo, J. Jaramillo, R. Gomperts, R. E. Stratmann, O. Yazyev, A. J. Austin, R. Cammi, C. Pomelli, J. W. Ochterski, P. Y. Ayala, K. Morokuma, G. A. Voth, P. Salvador, J. J. Dannenberg, V. G. Zakrzewski, S. Dapprich, A. D. Daniels, M. C. Strain, O. Farkas, D. K. Malick, A. D. Rabuck, K. Raghavachari, J. B. Foresman, J. V. Ortiz, Q. Cui, A. G. Baboul, S. Clifford, J. Cioslowski, B. B. Stefanov, G. Liu, A. Liashenko, P. Piskorz, I. Komaromi, R. L. Martin, D. J. Fox, T. Keith, M. A. Al-Laham, C. Y. Peng, A. Nanayakkara, M. Challacombe, P. M. W. Gill, B. Johnson, W. Chen, M. W. Wong, C. Gonzalez and J. A. Pople, Gaussian 03, Gaussian(version 6.1), Inc., Wallingford CT, 2004.

31 A. D. Becke, J. Chem. Phys., 1997, 107, 8554-8560.

32 E. Ruiz, A. Rodríguez-Fortea, J. Tercero, T. Cauchy and C. J. Massobrio, Chem. Phys., 2005, 123, 074102.

33 G. M. Sheldrick, SADABS, A Program for Empirical Absorption Correction of Area Detector Data, University of Göttingen, Germany, 1997.

34 G. M. Sheldrick, SHELXS-2014/7, Program for Crystal Structure Solution, University of Göttingen, Germany2014.

35 G. M. Sheldrick, SHELXL-2014/7, Program for Crystal Structure Refinement, University of Göttingen, Germany2014.

36 P. S. Kalsi, Spectroscopy of Organic Compounds, New Age International, New Delhi, 2008.

37 I. Mohammed-Ziegler and A. Grün, Spectrochim. Acta, Part A, 2005, 62, 506-517.

38 I. Mohammed-Ziegler, A. Hamdi, R. Abidi and J. Vincens, Supramol. Chem., 2006, 18, 219-234.

39 B. Dolenský, R. Konvalinka, M. Jakubek and V. Král, J. Mol. Struct., 2013, 1035, 124-128.

40 S. Mandal, G. Das and H. Askari, Struct. Chem., 2014, 25, 4351.

41 L. J. Bellamy, The Infrared Spectra of Complex Molecules, Wiley, New York, 1958. 
42 G. Świderski, A. Z. Wilczewska, R. Świsłocka, M. Kalinowska and W. Lewandowski, J. Therm. Anal. Calorim., 2018, 134, 513-525.

43 Y. Gong, Z. Hao, J. L. Sun, H. F. Shi, P. G. Jiang and J. H. Lin, Dalton Trans., 2013, 42, 13241-13250.

44 Y. Q. Xu, D. Q. Yuan, B. L. Wu, L. Han, M. Y. Wu, F. L. Jiang and M. C. Hong, Cryst. Growth Des., 2006, 6, 1168-1174.

45 Y. J. Cui, Y. F. Yue, G. D. Qian and B. L. Chen, Chem. Rev., 2012, 112, 1126-1162.

46 X. P. Yang, S. Q. Wang, L. J. Zhang, S. M. Huang, Z. P. Li, C. R. Wang, T. Zhu and L. Bo, J. Mater. Chem. C, 2016, 4, 1589-1593.
47 B. C. Yuan, F. Wang, J. B. Tao, M. Li and X. P. Yang, Inorg. Chim. Acta, 2019, 490, 24-28.

48 O. Kahn, Molecular Magnetism, VCH Publishers Inc, New York, 1993.

49 L. Tang, F. Fu, J. J. Wang, L. J. Gao, D. D. Chao and Z. Wang, Polyhedron, 2015, 88, 116-124.

50 C. Beghidja, G. Rogez, J. Kortus, M. Wesolek and R. Welter, J. Am. Chem. Soc., 2006, 128, 3140-3141.

51 E. Ruiz, P. Alemany, S. Alvarez and J. Cano, Inorg. Chem., 1997, 36, 3683-3688.

52 A. Rodríguez-Fortea, P. Alemany, S. Alvarez and E. Ruiz, Inorg. Chem., 2001, 40, 5868-5877. 